\title{
Advanced thermosets from sulfur, renewable benzoxazine and ionones via inverse vulcanization
}

Ozan Bayram ${ }^{1}$, Baris Kiskan ${ }^{1, *}$, Emrah Demir ${ }^{2}$, Rezan Demir-Cakan ${ }^{2,3, *}$, Yusuf Yagci ${ }^{1,4 *}$

${ }^{1}$ Istanbul Technical University, Department of Chemistry, 34469, Maslak, Istanbul, Turkey.

${ }^{2}$ Institute of Nanotechnology, Gebze Technical University, 41400 Gebze, Kocaeli, Turkey

${ }^{3}$ Department of Chemical Engineering, Gebze Technical University, 41400 Gebze, Kocaeli, Turkey

${ }^{4}$ King Abdulaziz University, Faculty of Science, Chemistry Department, 21589, Jeddah, Saudi Arabia

*Corresponding Authors: (B. Kiskan), (R. Demir-Cakan), (Y. Yagci)

e-mail: kiskanb@itu.edu.tr

demir-cakan@gtu.edu.tr

yusuf@itu.edu.tr

Number of pages: 5

Number of Figures: 6

Number of Schemes: 1 


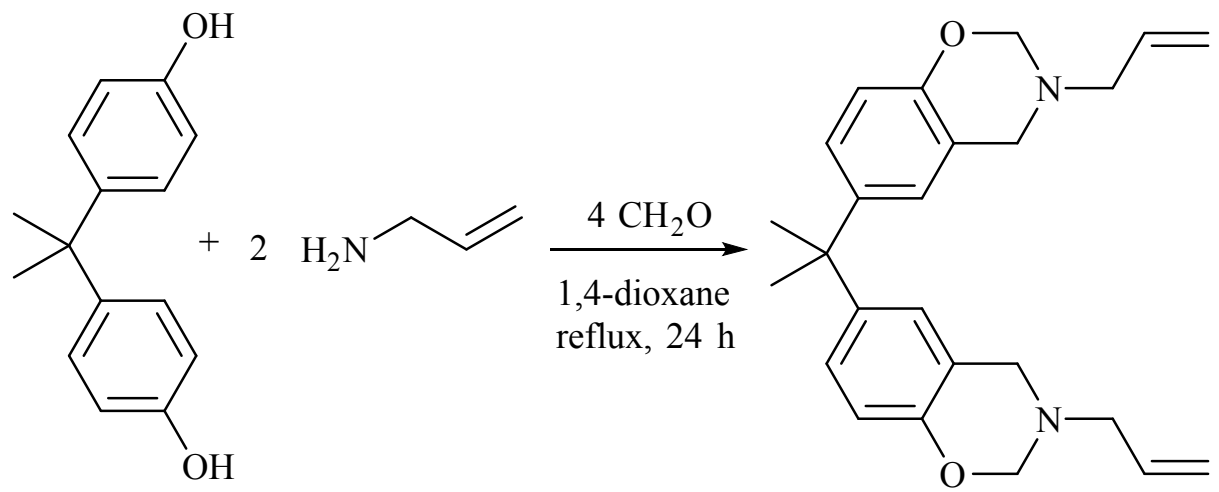

Scheme S1: Synthesis of bisphenol A based benzoxzine monomer (B-ala)

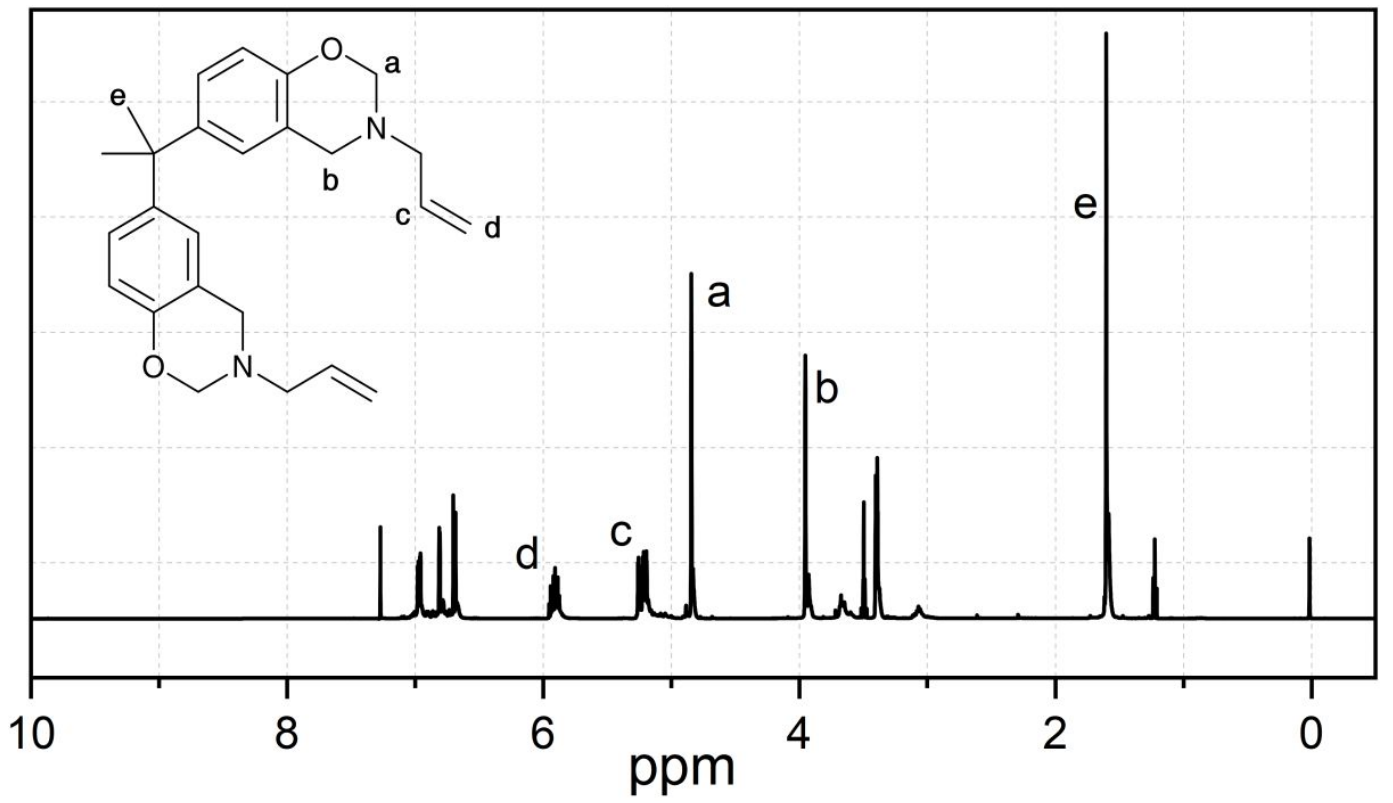

Figure S1: ${ }^{1} \mathrm{H}$ NMR spectrum of B-ala monomer 


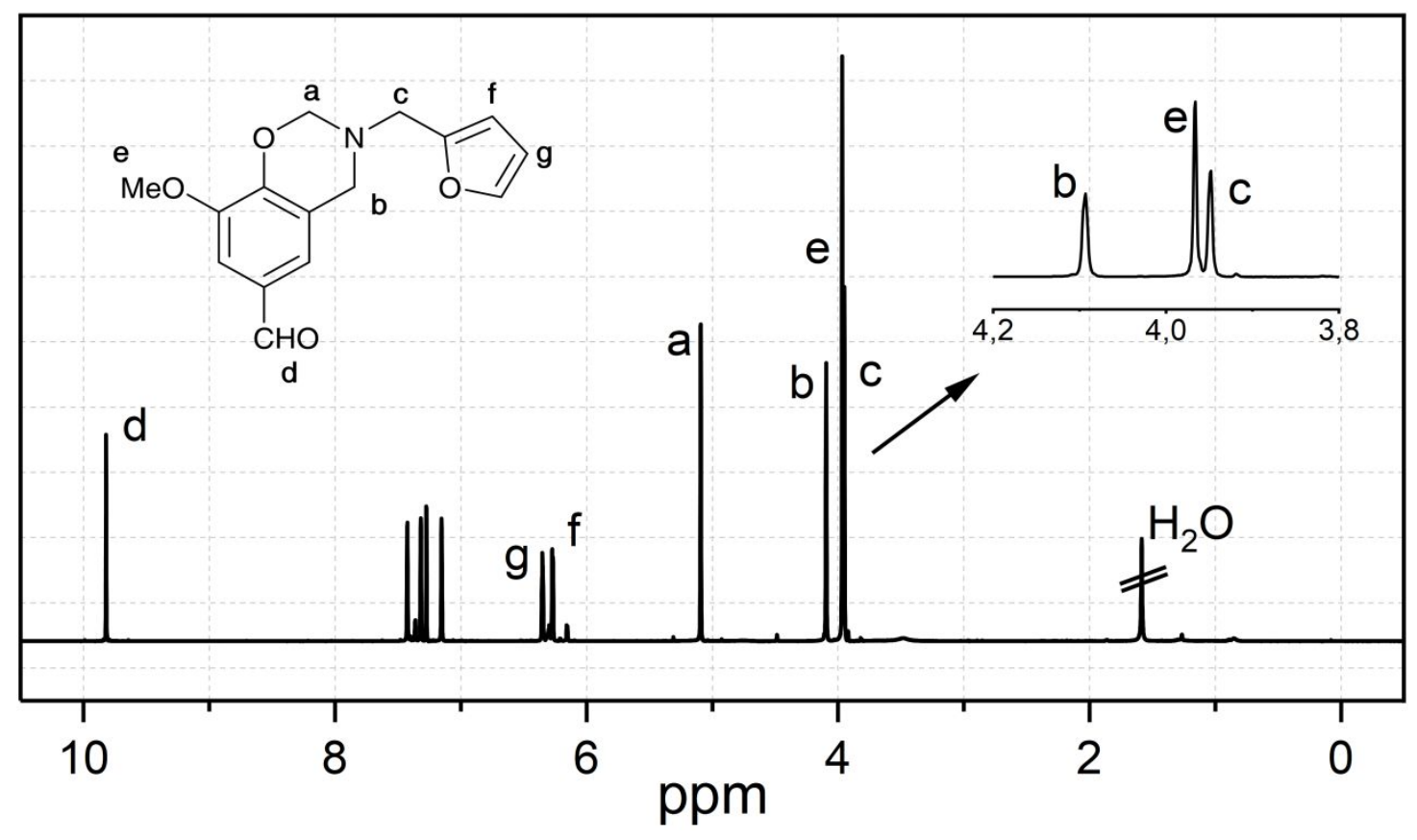

Figure S2: ${ }^{1} \mathrm{H}$ NMR spectrum of Va-Bz monomer

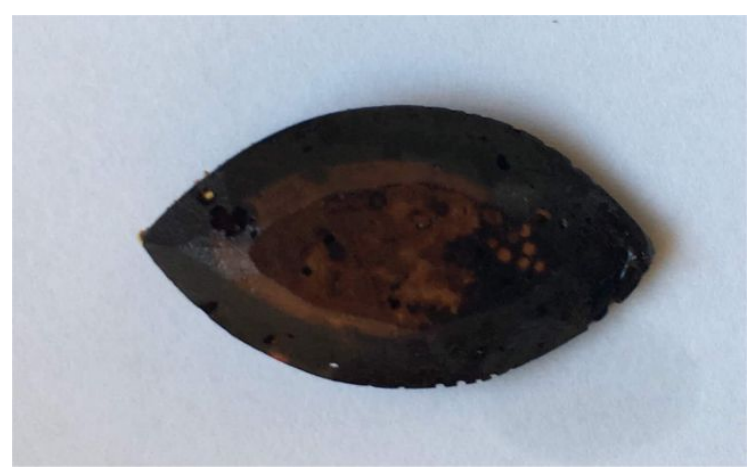

Figure S3: Image of molded poly(B-ala-S-ionone) 


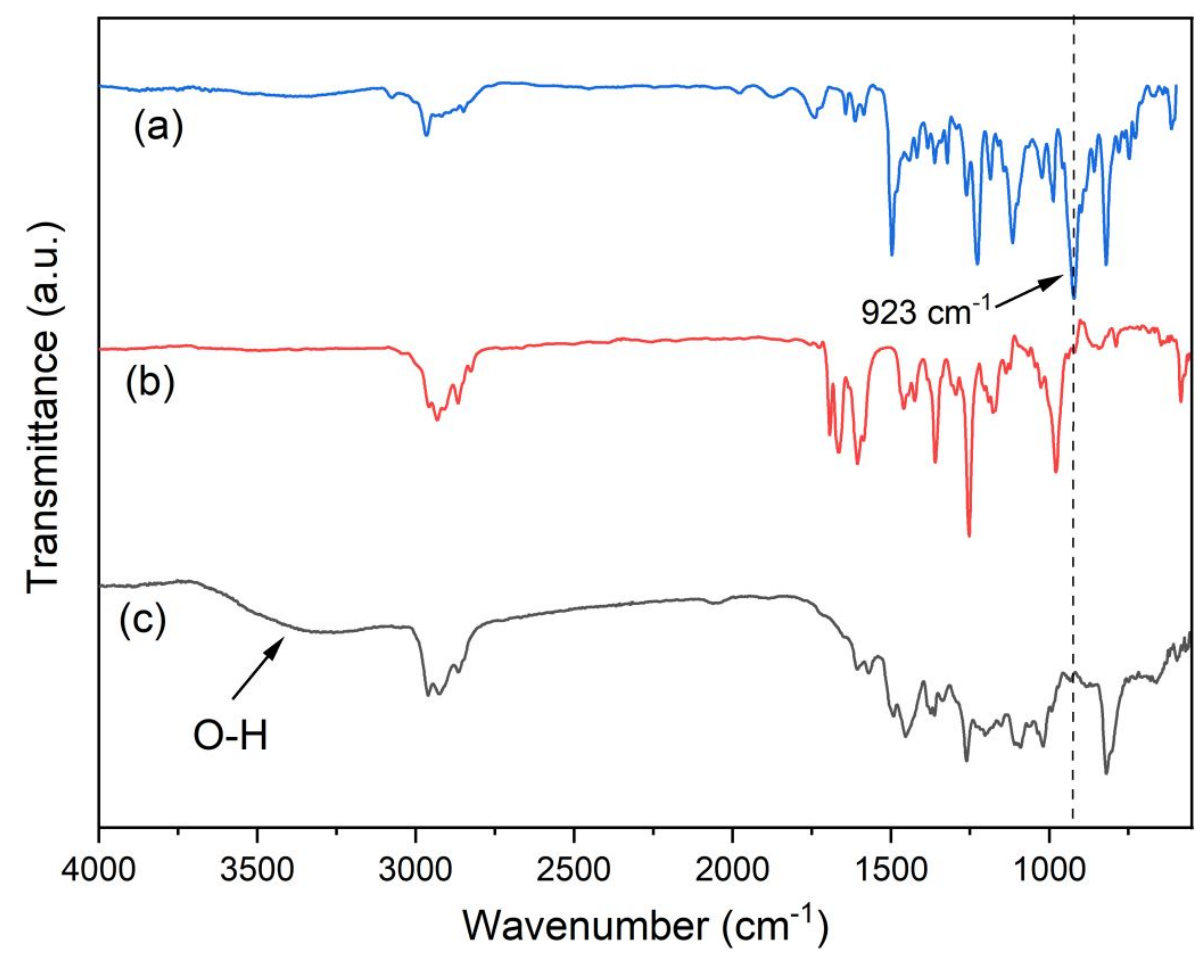

Figure S4: FTIR spectra of B-ala (a), $\beta$-ionone (b) and poly(B-ala $30-\mathrm{S}_{40}$-ionone $\left.{ }_{30}\right)$

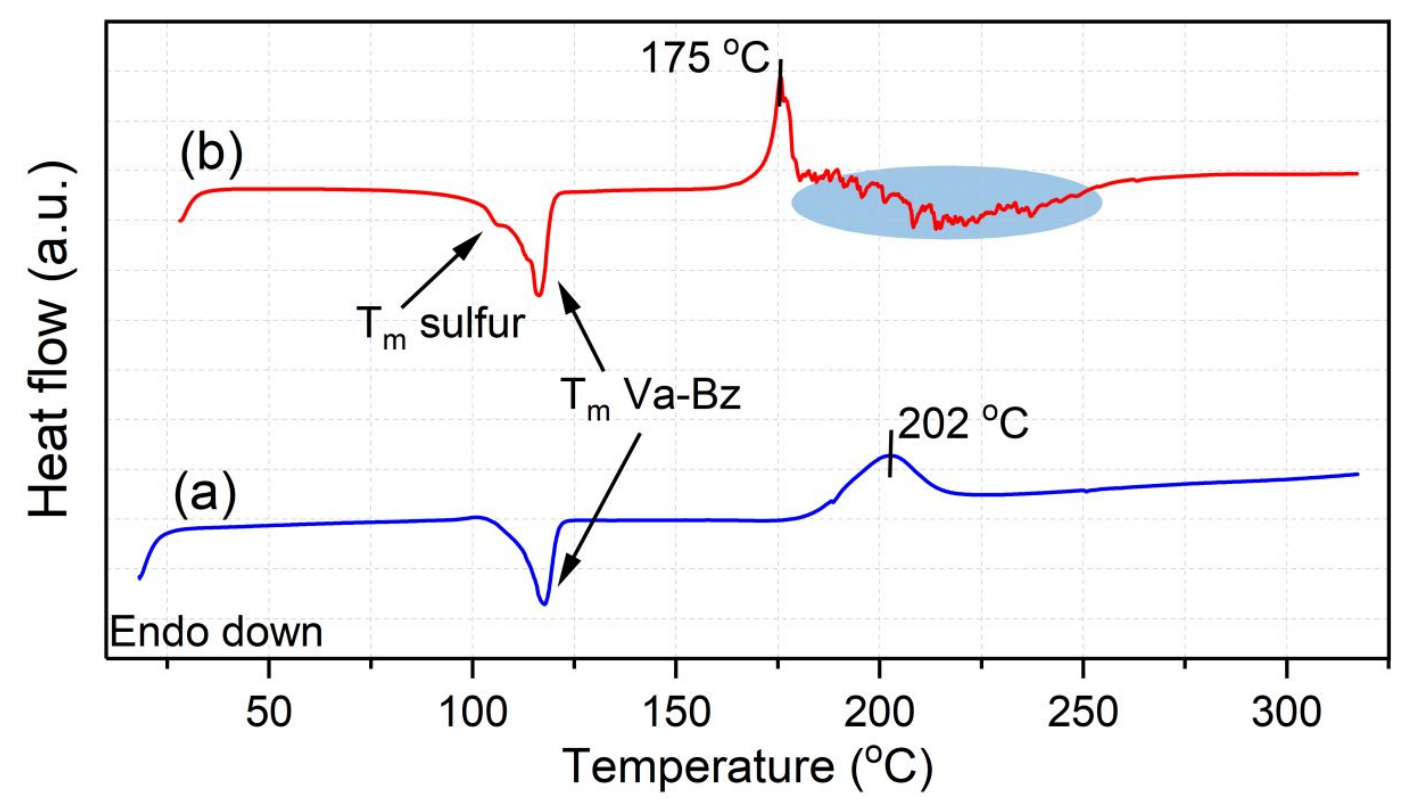

Figure S5: Overlaid DSC thermograms of Va-Bz (a) and Va-Bz/sulfur mixture (60:40, w/w) 


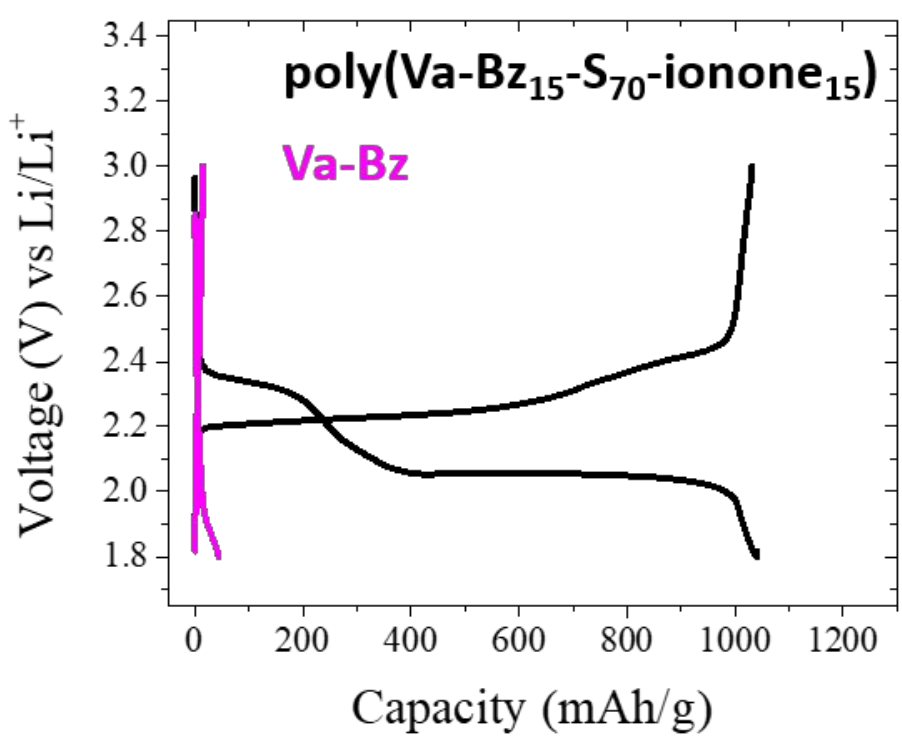

Figure S6: First galvanostatic discharge-charge profile of poly( $\mathrm{Va}-\mathrm{Bz}_{15}-\mathrm{S}_{70}$-ionone $\left.{ }_{15}\right)$ and Va-Bz cycled at $\mathrm{C} / 5$ current density 